\title{
El triángulo de la gestión energética en la empresa: optimización de compras, mantenimiento y eficiencia energética
}

\author{
Fernando Blanco Silva* \\ Universidad de Santiago de Compostela. La Coruña, España \\ Alfonso López Díaz* \\ Universidad Católica de Ávila. Ávila, España
}

Abel M. Venero Carrasco*

Estudio Santiváñez. Lima, Perú

Recibido: 25 de setiembre de 2013 / Aprobado: 16 de abril de 2014

Resumen: La energía es un componente del sistema de gestión en la empresa y uno de los aspectos más importantes de esta, como la gestión de recursos humanos o de compras. En el presente artículo describimos la gestión energética en el ámbito empresarial, la cual está definida por tres aspectos básicos: la optimización económica del gasto, el mantenimiento de las instalaciones y la implantación de medidas de eficiencia energética. Para conseguir una gestión óptima, es necesaria una solución que con el menor coste posible desempeñe los tres componentes de lo que hemos decidido llamar triángulo de la gestión energética.

Palabras clave: triángulo de la gestión energética / optimización de compras / mantenimiento y eficiencia energética

\section{The triangle of the energetic management in a company: optimization of purchases, maintenance and energy efficiency}

ABSTRACT: The energy is one of the components of management in the company, it is one of the main aspects of this management, such as human resource Department, the purchases or personnel. In this paper we describe the energy management, which is defined by three basic aspects such as economic optimization of purchases, the maintenance of facilities and the implementation of energy efficiency. To get the optimal management we need to optimize the three components of what we have decided to call the Triangle of Energy Management with the lower cost

Keywords: energy management triangle / optimizing purchase / maintenance and energy efficiency

* Correos electrónicos: fernando.blanco.silva@usc.es, alfonso.lopez@ucavila.es, abel.venero@santivanez.com.pe 


\section{INTRODUCCIÓN: LA IMPORTANCIA DE LA GESTIÓN ENERGÉTICA EN LA EMPRESA}

Consideramos la gestión energética como el conjunto de las tareas que se hacen en una empresa u organismo buscando optimizar los costes económicos derivados de su consumo de energía, entendiendo como tal encontrar una solución de compromiso que reduzca este consumo (y por lo tanto los costes energéticos) con la mínima inversión posible. En este concepto de gestión de la energía, existen otros aspectos indirectos como garantizar la prevención de riesgos laborales de los trabajadores que realizan las tareas de mantenimiento de las instalaciones (y por extensión de todos los trabajadores y usuarios del organismo), así como minimizar el impacto ambiental derivado de los consumos de energía; este impacto ambiental puede deberse al propio uso de recursos (electricidad, gasóleo, gas natural, biomasa, etcétera) o de las tareas de mantenimiento (aceites, cenizas, chatarras, residuos, entre otros).

La gestión en la empresa es un concepto global que aborda todos los procesos que tienen lugar en esta; para conseguir la excelencia, se deben aplicar las herramientas habituales de gestión como son el control de costes, optimización de recursos humanos, implantación de sistemas de gestión de la calidad o los sistemas informáticos de apoyo (SernaMachado, 2010). La persona que realiza estas tareas recibe el nombre de gestor energético y su formación debe ser técnica o universitaria (ingeniero o ingeniero técnico, preferiblemente de la rama industrial) e incluir los siguientes aspectos fundamentales:

1. Suministro de energía a menor precio. Las fuentes de energía que van a utilizarse en un edificio son la electricidad, el gas natural y derivados petrolíferos (gasóleo, fuelóleo y, en un sentido más amplio, incluso la gasolina o biocarburantes usados en transporte).

2. Mantenimiento de las instalaciones. Al menos se debe realizar el mantenimiento a las instalaciones eléctricas (en alta y baja tensión), calefacción, aire acondicionado, suministro de gas natural a generadores de calor (calderas), ascensores, barreras de aparcamiento, gases industriales o maquinaria industrial.

3. Eficiencia energética. Busca la realización de reformas sobre las instalaciones, sustitución de fuentes de energía o implantación de las fuentes renovables. 
Estos tres aspectos los podemos considerar el triángulo de la gestión energética, y la optimización de la empresa pasa por aunar actuaciones en dichos aspectos. Además, debemos considerar otro parámetro intrínseco, como es garantizar la prevención de riesgos laborales de las personas que trabajan en esta.

Figura 1

Triángulo de la gestión energética

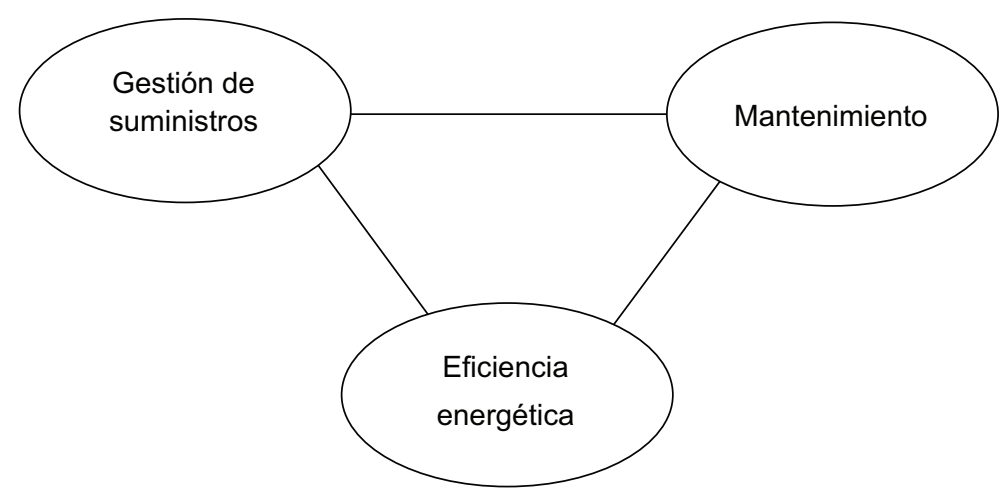

Elaboración propia.

Durante las últimas décadas, todos los países han implantado políticas energéticas con el fin de ajustarse a su realidad socioeconómica y obtener resultados concretos respecto de, por ejemplo, el acceso universal a la energía eléctrica, la seguridad energética, la competitividad de precios de la electricidad. En ese sentido, hasta la década de los noventa, los países desarrollaban planes energéticos nacionales cuya intención era diseñar las líneas básicas de la evolución de sus consumos de energía, aunque en general no eran documentos vinculantes y los resultados solían diferir de los objetivos marcados.

La aprobación del Protocolo de Kioto de 1997 introduce cambios importantes en esta dinámica, ya que se desarrolla el control de las emisiones de gases de efecto invernadero (GEI) que provocan el cambio climático y el calentamiento global. Entre estos gases de efecto invernadero, las emisiones de $\mathrm{CO}_{2}$ producidas por las combustiones de hidrocarburos, gas natural y biomasa suponían el mayor porcentaje. El 
Protocolo de Kioto generó la preocupación de todo el planeta por reducir las emisiones de GEI y, como consecuencia de este, todos los países hicieron una apuesta por fuentes que emitiesen menos $\mathrm{CO}_{2}$ (renovables, biomasa o gas natural) en detrimento de los derivados petrolíferos, aunque la intensidad de esta es muy diversa. Esta preocupación es gubernamental, los propios ministerios de Energía o Industria lideraban los esfuerzos para las reducciones del consumo de energía (Martínez, 2004; Lara, 2007). En los casos de España y Perú, los esfuerzos fueron, en mayor o menor medida, similares y congruentes con el resto de los países del mundo; no obstante, una particularidad en ambos países ha sido el esfuerzo por reducir la dependencia energética del exterior.

En el caso de España, la falta de recursos propios ha sido un problema histórico en la primera década del siglo XXI, el cual ha llegado a ser decisivo para el incremento de los precios del petróleo, y por extensión del gas natural. Desde el principio del presente siglo, el precio del barril se ha incrementado de forma sostenida, lo que ha provocado un encarecimiento continuo de la energía, por lo que es la unidad de consumo (doméstica o empresarial) la que se preocupa por reducir sus costes; estos van a ser, como norma general, el producto de la energía consumida (medida en $\mathrm{kWh}$ ) por el precio del material (medida en unidades monetarias/kWh), de manera que la solución pasa por reducir la cantidad de energía consumida (en $\mathrm{kWh}$ ) u optar por fuentes más baratas en términos unitarios (Estrada \& Hernández, 2009; Fernández et al., 2012).

Para el caso peruano, la presencia de un importante potencial hidroeléctrico e hidrocarburífero - básicamente gas natural, cuyas reservas han tenido importantes crecimientos en años previos (Saldani, 2006) — ha incrementado el interés en establecer una política energética que permita la inversión en dichos sectores, de manera tal que se asegure, primero, el aprovechamiento sostenible del potencial existente $\mathrm{y}$, segundo, precios razonables para los consumidores peruanos.

Partiendo del encarecimiento continuo de los combustibles tradicionales (petróleo y gas natural) y la apuesta por la reducción de gases de efecto invernadero, la política energética española, en mayor medida, y la peruana, en aspectos puntuales, han seguido las líneas estratégicas de los países oleodependientes (Isbell, 2006; Fontaine, 2009; Blanco, 2003), las cuales se podrían resumir en: 
- Ahorro de la demanda de energía útil.

- Mejorar la eficiencia energética y, por lo tanto, disminuir la demanda de energía primaria.

- Fomentar las fuentes autóctonas, en el caso de España estas son las fuentes renovables, mientras que en el Perú a estas fuentes se les suma el gas natural; las cuales también se fomentan con el fin de disminuir el impacto ambiental del consumo de energía.

- Evitar la dependencia del petróleo fomentando el gas natural y las fuentes renovables como energías sustitutivas.

Estas líneas estratégicas son objetivos nacionales pero surge un problema en la implicación por parte de la unidad de consumo (empresa o familia). Es obvio que para estos dos países sería muy positivo avanzar en cualquiera de los cuatro objetivos, no obstante, tanto las familias como los empresarios tienen como interés prioritario su propia economía, mientras que la conciencia ambiental o estratégica de su país funge como factor secundario en sus decisiones o - en la gran mayoría de casos- no amerita mayor atención.

\section{OPTIMIZACIÓN ECONÓMICA DE SUMINISTROS}

En este apartado incluimos básicamente las estrategias de gestión de compras que tiene la empresa, en las que debe existir una colaboración entre el departamento de compras y el gestor energético. En los países iberoamericanos, el suministro de energía se ha concebido como un servicio público y es muy intervenido por parte de la administración, con una casi inexistente competencia entre empresas, aunque esto va cambiando poco a poco y se está pasando de un monopolio a un oligopolio o competencias imperfectas que permiten pequeñas diferencias entre precios cuando hay un consumo muy elevado. La gran ventaja de esta optimización es que el coste de negociación es bajo (en muchos casos se limita a calcular las necesidades energéticas y solicitar precios a los proveedores), y los ahorros son inmediatos e indiscutibles si partimos de una situación inicial precio-aceptante.

En sentido estricto, incluiríamos en este grupo la compra de energía eléctrica, gasóleo o gas natural aprovechando factores de escala (compra directa a los generadores eléctricos o grandes distribuidores de hidrocarburos, compra en el mercado diario en el caso de grandes con- 
sumos o la comparación de precios entre diferentes comercializadoras cuando estos son más moderados, etcétera) y en un sentido más amplio también se puede incluir la compra de herramientas y consumibles (materiales eléctricos, térmicos y diferenciales, tuberías, entre otros implementos) cuando en el equipo de trabajo se incluyen operarios o la contratación de tareas externas de mantenimiento y rediseño. En este ámbito (tareas de mantenimiento y rediseño), es importante discriminar en qué casos se piden precios a empresas externas porque la definición completa de la actividad requiere en muchos casos la elaboración de un proyecto o memoria técnica, con unos costes de ingeniería que pueden llegar a ser elevados y en muchos casos no compensan el ahorro conseguido; en esta línea es muy recomendable contar con proveedores estables que por fidelización con el cliente van a presentar precios ajustados sin que sea necesario estar continuamente negociando, ya que el ahorro conseguido puede superar de largo los costes de gestión.

\section{MANTENIMIENTO DE LAS INSTALACIONES}

El segundo componente del triángulo no produce resultados tan inmediatos como la optimización de las compras, aunque mediante su correcta gestión sí hay un beneficio económico claro. Se trata de optimizar el funcionamiento de las instalaciones mediante un correcto mantenimiento; así, aseguramos el alargamiento de la vida útil de las instalaciones y garantizamos un funcionamiento sin interrupciones, además de mantener un elevado valor en el rendimiento durante esta vida útil.

Básicamente existen tres modalidades de mantenimiento: preventivo, correctivo y rediseño (o mejorativo). El mantenimiento preventivo incluye la relación de tareas por realizar antes de que suceda el fallo de la instalación; puede ser de dos tipos: programado (según un número de horas, número de ciclos) o predictivo (también llamado a condición, según la experiencia del técnico o pequeños fallos que anticipen uno más grave). El mantenimiento correctivo incluye la realización de tareas posteriores al fallo, que puede ser un fallo súbito (imposibilita el funcionamiento de toda la instalación dejándola fuera de servicio durante un periodo largo) o menor (afecta de forma considerable, pero permite un funcionamiento precario). El rediseño o mantenimiento mejorativo incluye cambios importantes en la instalación, y puede considerarse una mejora de eficiencia energética de las instalaciones. 
Es habitual que las empresas intenten minimizar los costes de mantenimiento limitándose a las tareas a las que obligan los reglamentos industriales al menor coste posible; esto puede originar problemas graves como el funcionamiento por debajo de los rendimientos habituales y el fallo súbito de la instalación. Existen obligaciones de mantenimiento para todas las instalaciones industriales, a título no exhaustivo citamos las recogidas en electricidad (reglamentos de baja o alta tensión, reglamentos de alumbrado), instalaciones térmicas (reglamentos de instalaciones térmicas en los edificios), ascensores, grupos electrógenos de emergencia, prevención de la legionella, instalaciones y depósitos de derivados petrolíferos, grúas torre y grúas móviles, compresores y otros aparatos a presión (en particular calderas), tanques de propano, instalaciones fotovoltaicas o de energía solar térmica, instalaciones contra incendios y en un sentido más amplio los vehículos o maquinaria industrial.

Un aspecto muy importante en la gestión del mantenimiento es distinguir entre la revisión y la inspección. La primera está a cargo de la empresa mantenedora o del propio usuario (cuando cuenta con el personal y los medios técnicos necesarios) y corresponde a las tareas de mantenimiento preventivo anual que garantizan el mejor funcionamiento posible, entendiendo como tal la optimización de su eficiencia, y la seguridad de que no se vaya a producir un fallo súbito. Las inspecciones son las tareas de mantenimiento programado (excepcionalmente es mantenimiento correctivo cuando ha habido algún accidente de cierta entidad) que garantizan el funcionamiento seguro de las instalaciones; no buscan la eficiencia, aunque la existencia de una deficiencia en la seguridad provoca siempre una pérdida de rendimiento energético.

En España, en los últimos años, se han introducido inspecciones que buscan garantizar rendimientos energéticos mínimos como son las de eficiencia energética en instalaciones térmicas, calderas o en cogeneración (aunque estas últimas son excepcionales). Las inspecciones son realizadas por la administración pública o por organismos colaboradores de esta, que en España reciben el nombre de organismos de control autorizado (OCA). Los organismos de control deben ser acreditados por la Entidad Nacional de Acreditación (ENAC) y deben demostrar su independencia de la empresa que los contrata, así como la solvencia técnica y económica (aval, seguro de responsabilidad civil, etcétera). 
Por otra parte, en el Perú, el año 2006 se emitió el Código Nacional de Electricidad. Utilización, que implicó una actualización general de las normas que regulan el uso de materiales y equipos eléctricos. Esta actualización trajo consigo la implementación de inspecciones iniciales y periódicas de las instalaciones eléctricas que serán realizadas por las autoridades competentes o por los inspectores autorizados y acreditados por estas.

Cabe señalar que este nuevo cuerpo normativo complementó las disposiciones existentes en el Código Nacional de Electricidad. Suministro, que se aplican a la infraestructura eléctrica involucrada en el suministro de electricidad y que no han tenido un desarrollo equiparable respecto de la regulación sobre inspecciones y revisiones de la infraestructura eléctrica.

Además de las revisiones e inspecciones, hay otro punto fundamental: la inscripción de las instalaciones en los correspondientes registros públicos, dependientes de la administración.

En España, prácticamente todas las nuevas instalaciones eléctricas (en alta y baja tensión) deben ser registradas ante la administración (Departamentos Autonómicos de Industria), mientras que en el campo de las instalaciones térmicas se deben registrar aquellas con potencias superiores a los $5 \mathrm{~kW}$; otras instalaciones que precisan de inscripción obligatoria son los pararrayos, las instalaciones susceptibles de legionella (torres de refrigeración, condensadores evaporativos, instalaciones de agua caliente sanitaria), los ascensores y los depósitos de derivados petrolíferos.

En el Perú no se ha logrado una diferenciación en el registro de las instalaciones eléctricas que vayan a ser instaladas; todas deben estar consideradas únicamente en la declaratoria de fábrica de las edificaciones.

Es especialmente importante reivindicar la vinculación del mantenimiento preventivo con la explotación energética. La realización de estas tareas suele ser obligatoria por normativa, pero el gestor debe transmitir al empresario la conveniencia de hacerlo; los costes de un buen mantenimiento son habitualmente mucho más reducidos que los costes de las grandes mejoras (cambios de calderas, renovaciones de alumbrados, entre otras) y una buena gestión —además de garantizar la seguridad del usuario- nos asegura un consumo razonable, 
un buen rendimiento energético eficiente y el alargamiento de la vida útil de las instalaciones, aunque es un hecho que muy a menudo pasa desapercibido.

Cuando tenemos noticia de problemas de legionella, accidentes eléctricos, incendios, incumplimientos de la prevención de riesgos laborales (PRL), estos suelen deberse al incumplimiento de la normativa en inspecciones; aunque el riesgo cero no existe en el caso de cumplirse estrictamente toda la normativa de las inspecciones, se evitaría un altísimo porcentaje de accidentes, además se tendría un control energético y, en el caso de producirse un accidente, las consecuencias serán mucho menos graves.

Para garantizar la seguridad de las instalaciones es bueno reforzar el control por las administraciones, estas además de ordenar la normativa deben realizar las tareas de supervisión, sancionando aquellas situaciones en las que se incumpla. En algunos casos hay propietarios o usuarios que no registran instalaciones para evitar el cumplimiento del régimen de inspecciones y revisiones.

Esto, que a priori supone un "ahorro", puede tener un efecto boomerang en un mal funcionamiento sostenido (falta de eficiencia) que en el caso de un desenlace fatal tendría una sanción muy severa, por ejemplo, si una vez acontecido un accidente grave o muy grave se demuestra que una instalación ni siquiera estaba registrada, la sanción será muchísimo más grave que aquellas registradas en las que no se cumplía la normativa; en el caso de instalaciones registradas y en las que se cumple la normativa es imprevisible la aparición de sucesos por la inexistencia del riesgo cero; la penalización será muy inferior a los dos casos anteriores.

A modo indicativo, presentamos a continuación una tabla que resume el régimen de inspecciones y revisiones de algunas instalaciones en España y el Perú. 
Tabla 1

Relación no exhaustiva de revisiones e inspecciones en algunas instalaciones energéticas en edificios en España y el Perú

\begin{tabular}{|c|c|c|c|}
\hline Tipo de instalación & Revisiones & Inspección & Normativa española \\
\hline $\begin{array}{l}\text { Eléctrica en baja } \\
\text { tensión }\end{array}$ & $\begin{array}{l}\text { Mensuales } \\
\text { (diferenciales) }\end{array}$ & Cada cinco años & $\begin{array}{l}\text { Reglamento Electrotécnico } \\
\text { de Baja Tensión (R. D. } \\
\text { 842/2002) }\end{array}$ \\
\hline $\begin{array}{l}\text { Eléctrica en alta } \\
\text { tensión }\end{array}$ & Anual & Trianual & $\begin{array}{l}\text { Reglamento sobre condicio- } \\
\text { nes técnicas y garantías de } \\
\text { seguridad en centrales eléc- } \\
\text { tricas, subestaciones y cen- } \\
\text { tros de transformación (R. D. } \\
3275 / 1982 \text { ) }\end{array}$ \\
\hline Ascensores & Mensual & $\begin{array}{l}\text { Cada dos, cuatro } \\
\text { o seis años, según } \\
\text { su uso }\end{array}$ & $\begin{array}{l}\text { Reglamento de aparatos de } \\
\text { elevación y manutención } \\
\text { (R. D. 2291/1985) }\end{array}$ \\
\hline Calderas & $\begin{array}{l}\text { Mensual, anual } \\
\text { y con otras fre- } \\
\text { cuencias, según } \\
\text { potencias }\end{array}$ & $\begin{array}{l}\text { Cada tres o cinco } \\
\text { años, según } \\
\text { potencias }\end{array}$ & $\begin{array}{l}\text { Reglamento de Instalaciones } \\
\text { Térmicas en los Edificios } \\
\text { (R. D. 1027/2007) y Regla- } \\
\text { mento Aparatos a Presión } \\
\text { (R. D. 2060/2008) }\end{array}$ \\
\hline $\begin{array}{l}\text { Grúas torre } \\
\text { para obras }\end{array}$ & $\begin{array}{l}\text { Semanal, quin- } \\
\text { cenal, mensual y } \\
\text { cuatrimestral }\end{array}$ & Cada dos años & $\begin{array}{l}\text { Instrucción Técnica Com- } \\
\text { plementaria } 2 \text { del Regla- } \\
\text { mento de Aparatos de Ele- } \\
\text { vación e Manutención (R. D. } \\
\text { 836/2003) }\end{array}$ \\
\hline $\begin{array}{l}\text { Alumbrado interior, } \\
\text { solar térmica y } \\
\text { fotovoltaica }\end{array}$ & $\begin{array}{l}\text { Trimestral, se- } \\
\text { mestral o anual }\end{array}$ & & $\begin{array}{l}\text { Código Técnico de la Edifi- } \\
\text { cación. Documento Básico } \\
\text { de Ahorro de Energía (R. D. } \\
\text { 314/2006) }\end{array}$ \\
\hline $\begin{array}{l}\text { Grúas móviles } \\
\text { autopropulsadas }\end{array}$ & Semestral & $\begin{array}{l}\text { Cada uno, dos o } \\
\text { tres años según } \\
\text { la antigüedad de } \\
\text { la grúa }\end{array}$ & $\begin{array}{l}\text { Instrucción Técnica Com- } \\
\text { plementaria } 4 \text { del Regla- } \\
\text { mento de Aparatos de Ele- } \\
\text { vación y Manutención (R. D. } \\
\text { 837/2003) }\end{array}$ \\
\hline $\begin{array}{l}\text { Instalaciones } \\
\text { contra incendios }\end{array}$ & $\begin{array}{l}\text { Trimestral, se- } \\
\text { mestral, anual o } \\
\text { cada cinco años }\end{array}$ & $\begin{array}{l}\text { Cada dos, tres o } \\
\text { cinco años }\end{array}$ & $\begin{array}{l}\text { Reglamento de Instalaciones } \\
\text { de Protección contra incen- } \\
\text { dios (R. D. 560/2010) }\end{array}$ \\
\hline
\end{tabular}

(continúa) 
(continuación)

\begin{tabular}{|l|l|l|l|}
\hline Tipo de instalación & \multicolumn{1}{|c|}{ Revisiones } & \multicolumn{1}{|c|}{ Inspección } & \multicolumn{1}{c|}{ Normativa española } \\
\hline $\begin{array}{l}\text { Tanques de almace- } \\
\text { namiento petrolíferos } \\
\text { para consumo propio }\end{array}$ & Cinco o diez años & $\begin{array}{l}\text { Cada uno, dos, } \\
\text { cinco o diez años }\end{array}$ & $\begin{array}{l}\text { Instrucción Técnica Com- } \\
\text { plementaria ITC-MI-IP03 del } \\
\text { Reglamento de Instalaciones } \\
\text { Petrolíferas (R. D. 2085/1994) }\end{array}$ \\
\hline $\begin{array}{l}\text { Eficiencia energética } \\
\text { en cogeneración }\end{array}$ & - & Anual & $\begin{array}{l}\text { R. D. 661/2007 de fomen- } \\
\text { to de electricidad de origen } \\
\text { renovable }\end{array}$ \\
\hline $\begin{array}{l}\text { Eficiencia energética } \\
\text { de instalaciones tér- } \\
\text { micas de calor y frío }\end{array}$ & - & Cada cuatro años & $\begin{array}{l}\text { Reglamento de Instalaciones } \\
\text { Térmicas en los Edificios } \\
\text { (R. D. 1027/2007) }\end{array}$ \\
\hline
\end{tabular}

\begin{tabular}{|c|c|c|}
\hline Tipo de instalación & Inspecciones & Normativa peruana \\
\hline $\begin{array}{l}\text { Eléctrica en baja } \\
\text { tensión }\end{array}$ & \multirow{6}{*}{$\begin{array}{l}\text { Dependiendo del tipo de estableci- } \\
\text { miento, las instalaciones eléctricas } \\
\text { deben inspeccionarse con una re- } \\
\text { gularidad específica: } \\
\text { - Para locales de alta concurren- } \\
\text { cia, una (1) vez al año. } \\
\text { - Para locales comerciales, una } \\
\text { vez cada dos (2) años } \\
\text { - Para viviendas, una vez cada } \\
\text { cinco (5) años. }\end{array}$} & \multirow{6}{*}{$\begin{array}{l}\text { Código Nacional de } \\
\text { Electricidad. Utilización } \\
\text { (RM 037-2006-EM/VME) }\end{array}$} \\
\hline Ascensores & & \\
\hline $\begin{array}{l}\text { Grúas torre para } \\
\text { obras }\end{array}$ & & \\
\hline $\begin{array}{l}\text { Alumbrado interior, } \\
\text { solar térmica y } \\
\text { fotovoltaica }\end{array}$ & & \\
\hline $\begin{array}{l}\text { Grúas móviles } \\
\text { autopropulsadas }\end{array}$ & & \\
\hline $\begin{array}{l}\text { Instalaciones } \\
\text { contra incendios }\end{array}$ & & \\
\hline $\begin{array}{l}\text { Eléctrica en alta } \\
\text { tensión }\end{array}$ & $\begin{array}{l}\text { El equipo eléctrico será sometido a } \\
\text { inspección y mantenimiento en los } \\
\text { intervalos que, según la } \\
\text { experiencia, se consideren } \\
\text { necesarios. }\end{array}$ & $\begin{array}{l}\text { Código Nacional de } \\
\text { Electricidad. Suministro } \\
\text { (RM 366-2001-EM/VME) }\end{array}$ \\
\hline
\end{tabular}

Elaboración propia. 


\section{EFICIENCIA ENERGÉTICA}

Nos queda por analizar la tercera parte del triángulo: la eficiencia energética. En un sentido estricto, podemos considerar como tal la relación existente entre la energía realmente demandada y la energía primaria que se gasta para abastecer esta; el gestor debe considerar un concepto más amplio e incorporar el ahorro de energía (reducción de la demanda) o la incorporación de fuentes renovables y de otras poco contaminantes (en particular el gas natural cuando se utiliza como sustitutivo de los derivados petrolíferos). Existen miles de posibilidades para ahorrar dinero y/o energía en busca de la eficiencia energética; la función del gestor es discriminar cuáles son convenientes y cuáles no; el criterio que se usa es el periodo de retorno de la inversión y según este podemos clasificar las actuaciones en tres grupos:

a) Inversiones seguras. Básicamente son las que apenas tienen incertidumbres por ser muy estables tanto los consumos como los precios. En este grupo se incluirían tecnologías como la mejora de los alumbrados nocturnos y, en general, los que tengan un número de horas de funcionamiento anual elevado y previsible o la sustitución de derivados petrolíferos por gas natural o biomasa (combustibles en la actualidad más baratos). Se debe asegurar un periodo de retorno menor o igual a cinco años.

b) Inversiones inciertas. Se incluirán aquellas en las que existen importantes incertidumbres, los periodos de retorno son inciertos o demasiado largos (en general, por encima de los cinco años) de manera que el escenario energético se convierte en impreciso, y no se garantiza que la inversión sea conveniente.

c) Inversiones de carácter demostrativo. Se refiere a las inversiones en las que el gestor energético es consciente de que el periodo de retorno es demasiado largo, por lo que la decisión se tomará según las sinergias de la acción. Se incluyen en este grupo las docentes, experimentales, mejoras del confort en el interior de los edificios, las que implican alta publicidad para la empresa promotora, innovaciones y en general aquellas que con periodos de retorno superiores a diez años tienen sinergias que permiten otros aprovechamientos secundarios.

En general, estas actuaciones son bastante conocidas y las propuestas se multiplican por parte de gestores, consultorías, empresas eléctricas, constructoras que ofrecen sus servicios. En algunos casos, el periodo de retorno es claro (cambios de calderas, mejoras en alumbrados 
nocturnos, sectorización de edificios), mientras que en otros como la renovación de cerramientos, baterías de condensadores o saneamiento integral de instalaciones los ahorros son más discutibles.

\section{Fotografía 1}

Motor de cogeneración situado en la Universidad de Santiago de Compostela (España)

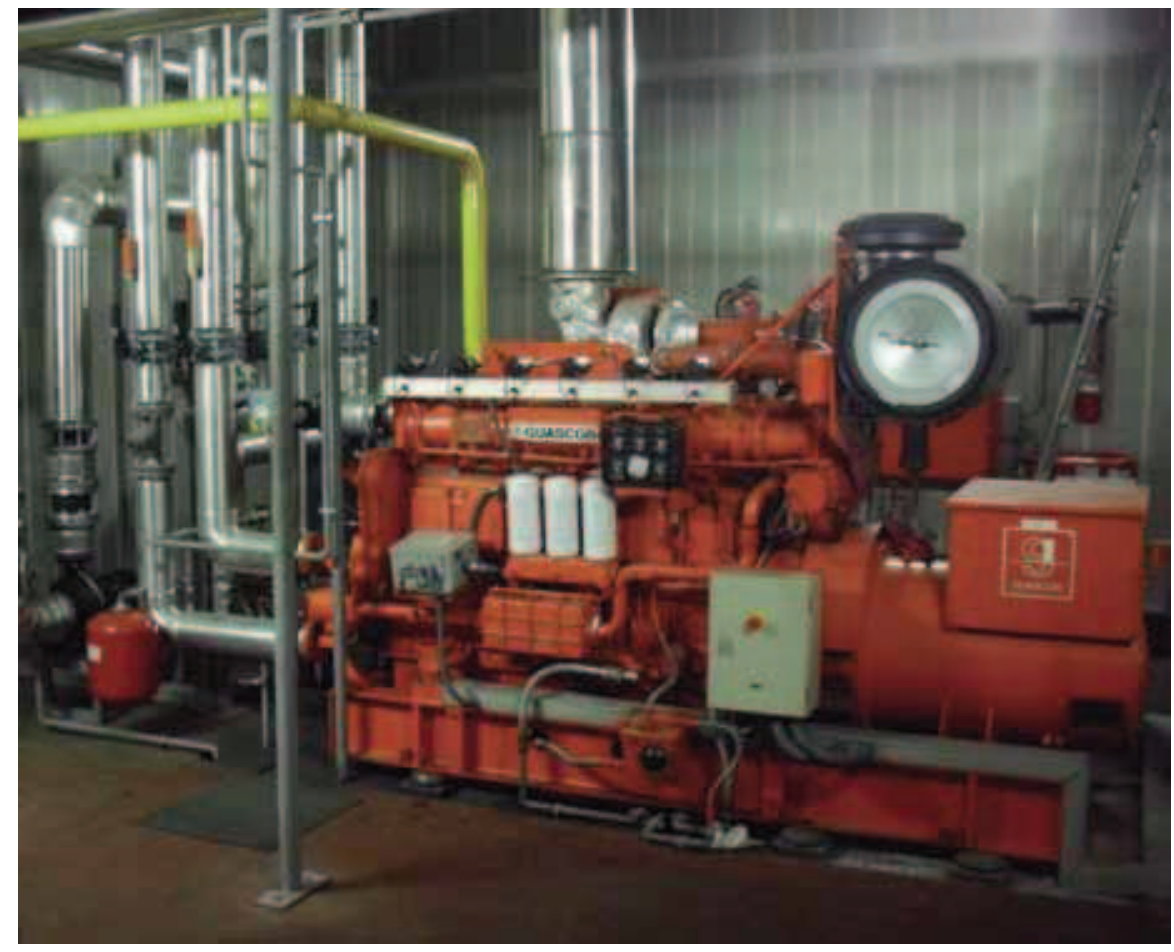

\section{OTROS ASPECTOS IMPORTANTES EN LA GESTIÓN ENERGÉTICA: LA PRESERVACIÓN DEL MEDIOAMBIENTE Y LA PREVENCIÓN DE RIESGOS LABORALES}

El gestor energético será la persona responsable de la realización de las principales tareas en las instalaciones que aquí hemos denominado triángulo de la gestión energética, aunque normalmente debe realizar otras tareas de control, como la ejecución de pequeñas obras o la reducción al mínimo del impacto ambiental en la propia explotación. 
Al implantar una mejora en las instalaciones (por ejemplo, el rediseño), establecer medidas de eficiencia energética o simplemente llevar a cabo el mantenimiento de estas, se van a realizar tareas que pueden ser consideradas como obras (aquellas que disponen de un proyecto de ejecución) u otras que sin disponer de proyecto no son tareas ordinarias y repetidas, por lo que se exige un esfuerzo adicional por parte de la persona que ejerce la gestión energética y que podemos afirmar que está desempeñando las funciones de director de obra. Entre las funciones de este se encuentran la de garantizar la prevención de riesgos laborales de todos los participantes (operarios), así como de las personas y los bienes afectados por estas tareas. Para ello, es necesario que el director de obra disponga de formación suficiente en prevención de riesgos laborales.

En España, los niveles de formación de riesgos laborales son tres: el curso básico de cincuenta horas, el ciclo formativo de grado superior (orientado a las personas que se dedican exclusivamente a estas funciones) y el máster universitario oficial de prevención de riesgos laborales; es necesario que el gestor energético haya cursado el curso básico de PRL (en la práctica, es una exigencia por parte del empresario que el gestor energético disponga de este título) y es muy recomendable que además curse la formación universitaria de máster oficial, porque suele desempeñar las funciones de coordinador de seguridad y salud en la fase de ejecución de la obra.

En cambio, en el Perú, dado que las normas generales referidas a seguridad y salud son recientes, la oferta educativa en formación de riesgos laborales aún no ha logrado la sofisticación comentada en España. No obstante, cada vez más los profesionales vienen requiriendo especialización en esta área, con lo que diversos tipos de cursos y diplomados relacionados empiezan a hacerse visibles.

Como último aspecto destacado está la responsabilidad ambiental de la empresa. En un momento en el que las memorias de responsabilidad social corporativa de las empresas transmiten a la sociedad una preocupación ambiental por parte de estas es necesario un técnico que gestione estas actuaciones. El gestor energético no tiene a priori una formación específica en medioambiente, aunque por su formación previa es habitual que además de sus funciones como gestor asuma las propias de un técnico medioambiental. 


\section{REFERENCIAS}

Estrada, A., \& Hernández, P. (2009). El precio del petróleo y su efecto sobre el producto potencial. Documentos ocasionales-Banco de España, 2, 11-29.

Fernández, E., Pérez, R., \& Ruiz, J. (2012). Análisis dinámico del impacto de los shocks en el precio del petróleo sobre el empleo por sectores productivos. Economía Industrial, 384, 85-98.

Folgado, J. (2003). La política energética en España. Información Comercial Española (ICE). Revista de Economía, 811, 29-33.

Fontaine, G. (2009). Las políticas energéticas de cara al desarrollo sostenible en Perú y Ecuador. América Latina Hoy, 53, 103-126.

Isbell, P. (2006). La dependencia energética y los intereses de España. Análisis del Real Instituto Elcano, 32(1).

Lara, M. de (2007). Cambio climático: una preocupación creciente. Economistas, 25(113), 78-85.

Martínez de Bascarán, G. (2004). El Protocolo de Kioto y sus consecuencias. Revista Ingeniería Química, 412, 178-182.

Saldani, A. (2010). Informe Sectorial Perú: Sector Hidrocarburos. Pacific Credit Rating. Recuperado de http://www.ratingspcr. com/archivos/publicaciones/SECTORIAL_PERU_ HIDROCARBUROS_201006.pdf

Serna-Machado, C. (2010). Gestión energética empresarial una metodología para la reducción de consumo de energía. Revista Producción + Limpia, 5(2), 107-126. 